\title{
La Ville et ses prosopopées fabuleuses
}

\author{
Nathalie Roelens \\ "The city is an addictive machine from which there is no escape" (La métropole est une machine qui agit comme une \\ drogue et à laquelle il est impossible d'échapper : Rem Koolhaas 293)
}

\section{INTRODUCTION}

Afin d'exorciser ses peurs face à la ville, l'inconscient collectif l'a souvent revêtue de métaphores anthropomorphes, voire féminines, c'est-à-dire de prosopopées. Les artistes ou écrivains se substituent à l'urbaniste pour conférer une âme à un ensemble inanimé. Car en transfigurant la ville, ils la resémantisent, l'investissent d'un pouvoir magique. C'est en quelque sorte le moteur de la "pensée utopique" (Trousson, Voyages 16).

Nous verrons cependant que la ville mythique s'avère essentiellement éphémère, que l'illusion euphorique se mue en mirage dysphorique, que les utopies "dégénèrent” pour utiliser une formule de Louis Marin (Marin, "Dégénérescence" 31), comme si l'imaginaire humain avait une vocation à se méfier de ses propres élucubrations, comme si le principe de réalité reprenait toujours le dessus. L'imaginaire, une fois sédimenté en fiction, se voit privé de sa densité fabuleuse au profit de toutes sortes de récupérations idéologiques, qu’on les appelle “fantasmagorie” (Benjamin 47), "hallucination” (Marin, Dégénérescence 50), ou "simulacre" (Baudrillard 33).

L'opposition entre fiction et imaginaire qui se dégagera progressivement de nos propos nous semble opérationnelle car emblématique de ce qui se passe dans les sciences humaines où l'on assiste à un déclin d'une 
théorisation surplombante, friande de clôture fictionnelle et d'immanence, au profit d'une théorie plus soucieuse des pratiques de sens, des institutions discursives et de l'imaginaire des textes ou des images.

\section{VILLES MYTHIQUES}

Dans le récit fondateur de la Rome mythique c'est la séparation de la campagne environnante par le tracé de la charrue de Romulus qui prime, définissant ainsi le périmètre, réputé sacré et inviolable, de la future cité. Que ce sillon donne une assise épistémologique à toute prosopopée urbaine, Régis Debray nous le précise dans L'Éloge des frontières où il rend hommage à ces premiers constructeurs de villes qui ont avant tout configuré un site en traçant une ligne, en séparant l'autorisé de l'interdit. Ce "geste inaugural ... de démarcation" (Debray, Éloge 25) fait bien sûr allusion à l'augure qui définissait un templum (du grec temnein: couper) à des fins rituelles et pour la con-templation, un espace "découpé" comme sacré. Chateaubriand fait écho à ce mythe fondateur dans sa visite de la Villa d'Hadrien, passage obligé de son Voyage en Italie, lorsqu'il constate la nonchalance avec laquelle on a conservé ce patrimoine riche en ruines. "Les empires naissent de la charrue et disparaissent sous la charrue" (Chateaubriand 82) est la phrase que lui inspire le théâtre romain de la Villa, noble vestige de la Rome antique converti en ferme où broutent des chèvres. À Rome il assiste à une double décadence: celle de l'Empire romain et celle de l'Empire chrétien qui s'est érigé sur ses décombres. En effet, dans les premiers siècles de l’ère chrétienne, la ville devient métaphore du mal à laquelle s'oppose la spiritualité de la vie monastique selon 'l'opposition augustinienne entre la ville réelle, siège du vice, et la ville céleste, symbole de l'amour de Dieu"' (Battistini 266). De même, la Rome-louve nourricière peut se muer en Grande Prostituée, Babylone, mère corrompue et corruptrice.

Le Paris légendaire connait également cette oscillation entre cité mère archétypale, déesse tutélaire ou lieu de fécondité, et putain lascive. Ainsi la fougue métaphorique hugolienne dans Notre-Dame de Paris décline-telle la physionomie de l'Île de la Cité en une série de trompe-l’œil imagés: berceau, mère, navire, tortue tandis que la Seine est qualifiée de "nourricière" (Hugo 132). Dans “L’Orgie parisienne ou Paris se repeuple”, poème d'Arthur Rimbaud, on retrouve le mélange de sainteté et d'abjection. Le poète visionnaire juxtapose "la Cité belle, assise à l'occident" et "la rouge courtisane aux seins gros de Batailles" (Rimbaud). Les surréalistes appréhenderont la ville comme un "vaste corps urbain" (Gracq 95) avec une tour Eiffel qui fait office de 
"langue tirée aux Allemands" pour Apollinaire (Apollinaire) ou, ironiquement, d'organe masculin pour Queneau: "Je me demande pourquoi on représente la ville de Paris comme une femme. Avec un truc comme ça" (Queneau 89), s'exclame Gabriel dans Zazie dans le métro. Dans la Clé des champs André Breton, à son tour, comparera la place Dauphine à un sexe féminin car, par sa forme triangulaire délicate, elle évoque pour lui un pubis lové entre deux "cuisses" (Breton) de Seine, dont les courbes lui inspirent tout un érotisme féminin (fig. 1). Que ce soit l'écriture qui engendre la morphologie de l'image ou l'inverse, peu importe, l'imaginaire est en tout cas à l’œuvre dans cette réécriture/remodelage de la ville référentielle, dans ce que les tenants de la géocritique (l'étude des espaces humains dans les textes et les images) appellent "reconfiguration d'un réalème" (Westphal 169).

Naples, quant à elle, a toujours été dotée d’une prosopopée fémine, de voluptueuse sirène. On a toujours privilégié sa féminité, son "ventre," ses"entrailles" de mère-mer amniotique baignant les flancs "sensuels" du Vésuve. Jean-Noël Schifano entrevoit dans sa silhouette épousant le golfe "un grand oiseau de feu en piqué avec son bec vers la mer" (Schifano, Naples 45) et dans sa stratification urbaine un gâteau feuilleté, une "sfogliatella" (Schifano, Dictionnaire 445) blonde et charnelle, audacieuse mise en abyme culinaire digne de la madeleine de Proust.

On le voit, la sensualité, pour peu qu'elle ne se dégrade pas en débauche, semble plutôt indexer positivement une ville, lui garantir une densité charnelle. Aussi les villes féminines, sensuelles, semblent-elles moins enclines à "dégénérer" qu’une ville artificielle. C’est dans ce sens que Régis Debray, dans son ouvrage Contre-Venise cette fois, confronte Naples et Venise. La Naples "putassière, charnue, généreuse, effrontée" l'emporte dans son imaginaire sur une Venise aseptisée, "microcosme égocentrique", "simulacre", ou "villejouet" (Debray, Contre-Venise 29-32). Venise, par son autonomie politique et symbolique, par sa "coupure sémiotique" (Debray, Contre-Venise 32) du reste de l'Italie, davantage ville-musée que lieu de vie, nous mène ainsi à notre deuxième point: l'utopie.

\section{UTOPIES}

Tandis que les villes mythiques étaient éphémères par leur côté légendaire, les utopies sont vulnérables par leur aspect purement conceptuel, ayant une vocation à demeurer à l'état de brouillon, de matrice. C'est le système totalisant des utopies, qu'il soit social ou architectural, avec ses normes et ses lois immuables, un devoir-être 
régissant les comportements, qui les rattache inévitablement à un pouvoir panoptique, témoin la structure souvent concentrique qui permet la surveillance totale et l'uniformisation des consciences.

Raymond Trousson a bien montré que ce monde clos et parfait ne supporte pas l'intrusion du monde réel, ni aucune initiative privée (suspecte parce que réfractaire au collectivisme) et encore moins des arts (potentiellement dissidents). On le voit, l'urbanistique est indissociable du politique. La dystopie serait alors l'incarnation d'une utopie réalisée et dès lors dégénérée, car, comme le montre David Harvey, "le libre jeu de l'imagination" tant vanté par Louis Marin s'y voit bloqué "dans sa recherche d'alternatives" (Harvey 157).

L’utopie, ne veut-elle pas dégénérer, doit donc rester à l'état de projet. Souvent les toponymes participent de cette nature déviante par rapport au réel, de non-lieux, de "mondes impossibles" pour paraphraser en creux Umberto Eco (Eco 113): l'Abbaye de Thélème de Rabelais, l'Utopia de Thomas More, l'Eldorado de Voltaire, Les villes invisibles d'Italo Calvino, ou encore, le lexème "land" ou "city" se voit rajeuni ou féerisé par son épithète: le Nonveau monde amoureux de Charles Fourier, phalanstère utopique; New Babylon de Constant, projet d'utopie sociale dans les années '60 fondée sur l'Homo ludens; Dreamland, le parc d'attraction mégalomane inauguré en 1904 sur le site de Coney Island à New York et dont l'architecture du sensationnel se propagea dans la société des loisirs du monde entier; Neverland, l'île allégorique créée par James Matthew Barry pour son livre Peter Pan; la Cité radieuse, cette unité d'habitation sociale pilote se voulant exemplaire, édifiée en 1952 à Marseille par Le Corbusier, et cetera.

Les représentations du Paradis terrestre tendent quant à elles à exacerber la clôture et la coupure sémiotique. "Dans l'iconographie médiévale, suite à la renaissance de l’urbanisation, le Paradis, originairement représenté comme jardin, assumera les traits d'un centre urbain" (Battistini 266). Toutefois, ce trait urbanistique dénonce "l’orgueil prométhéen" (Trousson, "La cité" 5) de toute spéculation utopique au MoyenÂge. Le royaume du Christ “n'est pas de ce monde," rappelle saint Augustin (Saint Augustin CXV). L'homme doit aspirer à se libérer de la cité terrestre. D’où le motif de la Jérusalem céleste remontant à l'Apocalypse de saint Jean, lieu spirituel qui descend du ciel et resplendit de gloire.

Certaines Villes invisibles d'Italo Calvino pourraient encore rappeler la Jérusalem céleste ou Laputa, la ville volante des Voyages de Gulliver de Jonathan Swift (Swift chap.5), mais imprégnées de l'ironie tendre et amère de l'auteur. Les esquisses urbaines de Calvino sont tellement exotiques qu'elles s'avèrent improbables, relevant de l'ailleurs absolu. Ce sont des villes que Marco Polo a seulement fantasmées, désirées: le plan des rues de Zobéide fut construit comme une trappe destinée à capter une femme entrevue en rêve par les architectes (Calvino 59-60) et la physionomie de Zemrude change selon l'humeur de celui qui la regarde 
(Calvino 81). Or la fable utopique se fait chemin faisant de plus en plus pessimiste et infernale, la décadence et la mort remplaçant le désir. Les habitants de Bersabée, obsédés par une cité céleste qu'ils imaginent tout en or et en pierres précieuses, ne se rendent pas compte qu’ils sont en train de bâtir la Bersabée souterraine basée uniquement sur la soif du gain et comprenant les détritus et les matières fécales de la ville terrestre. Dans une promenade fictive à travers les villes métaphysiques et désincarnées de Giorgio de Chirico, Calvino racontera (en 1983) l'agoraphobie et ensuite la claustrophobie qu'il éprouve en tant que corporéité humaine refoulée de ces villes de l'esprit: invivables comme toutes les utopies (Roelens).

Toute perfection semble dès lors vouée à l'auto-destruction comme si celle-ci était inscrite dans l'utopie même. Louis Marin voit dans Versailles, à la suite de Félibien, “l’expansion, le déplacement métonymique"(Marin, Le Portrait 235) du corps glorieux du roi. L’irruption d'une "théophanie festive" (Marin, Le Portrait 240) en 1674 avec les six jours de fêtes, à l'instar de la Genèse biblique, signe cependant une limite: le dispositif spectaculaire doit, pour s'accomplir, s'achever le septième jour par l'anéantissement, l'embrasement final de la machine: "Le simulacre est retourné et reversé dans le réel même dont la consumation se représente en ultime spectacle" (Marin, Le Portrait 248). Cette exubérance autodestructrice s’était déjà vérifiée à Vaux-le-Vicomte dont Fouquet n'avait pas prévu le fiasco inhérent à la réception “trop somptueuse”: “Vaux, énorme échec pétrifié; mais ce n’est pas l'échec d’un fou, ce fut le décor d'une réussite parfaite, qui n'a duré qu'une seule soirée, celle du 17 août 1661” (Morand 87).

On distingue un même passage du ludique au dystopique dans les premiers parcs d'attraction si l'on considère que leur esthétique du collage et de la copie aurait servi de modèle et de norme pour l'urbanisme kitsch à venir: Dreamland comptait, parmi ses attractions, une balade sur les "canaux de Venise” bordés de façades en toile, ou encore la "destruction de Pompéi”. Rem Koolhaas a célébré cet exploit architectural comme "une sorte de Manhattan fœetal" (Koolhaas 30), un monde d’illusions dans lequel on a insufflé de la vie, mais qui rate toujours de réel. Le "délire" de New York se révèle précisément dans l'apothéose du quadrillage “au mépris de la topographie, c’est-à-dire du réel existant” (Bajac \& Ottinger 30).

Les quelques projets urbanistiques inaccomplis de villes idéelles échappent sans doute à cette dégénérescence car délibérément irréalisables, elles questionnent la raison d'être même de la ville: villes ludiques, gonflables, nomades, modelables, conçues pour un homo ludens libéré du travail et des espaces utilitaires de l’homo faber et s’inscrivant dans l'imaginaire situationniste des années ‘60: New Babylon, l’utopie égalitaire de Constant; l'aérienne et éphémère Instant City des architectes d’Archigram ville nomade, qui se déplace, élément par élément, héliporté par des dirigeables ou des montgolfières ; le projet de ville refusé par les instances 
religieuses londoniennes Fun Palace de Cedric Price, espace flexible et indéterminé susceptible d'accueillir plusieurs activités comme le théâtre, la danse ou la musique.

\section{DYsTOPIES}

On le voit, les utopies ont une vocation à dégénérer, dès qu'elles se collètent avec le réel. Que la dystopie soit essentiellement conditionnée par la réalité urbanistique, même la plus élémentaire, s’observe déjà dans la Sodome biblique dont la destruction est imputable davantage à la clôture de la ville, au manque d'hospitalité des habitants, qu’à leurs mœurs inverties. Dans Métropolis de Fritz Lang, donjon moderniste déshumanisé et cauchemardesque, dans Tativille de Jacques Tati, la ville futuriste avec ses immeubles de verre et d'acier, froids et impersonnels qu'il a conçue pour son film Playtime, dans la Syldavie d'Hergé, le pays imaginaire dictatorial situé en Europe de l'Est évoqué dans Le sceptre d'Ottokar, ce sont les dysfonctionnements, la stratification sociale ou la bureaucratie qui transforment celles-ci en manifestes diaboliques. Derrida avait déjà attribué l'effondrement de l'utopie marxiste ou fasciste au fait qu'elle se soit muée en idéologie, à son inhospitalité foncière et à l'inscription dans le réel d'un lieu et d'un pouvoir lié à “la logique implacable de l'efficiency économique qu'elle prétendait combattre” (Derrida 22).

Louis Marin a, mieux que quiconque, décrit Disneyland comme un "exemple de dégénérescence de la pratique dans son produit" (Marin, "Dégénérescence" 31) avec son axis mundi Main Street USA, qui dirige le visiteur "de la réalité au fantasme” (Marin, "Dégénérescence” 36). C’est cette aliénation de l’usager qui nous pousse à inclure Disneyland dans les dystopies: l'imaginaire s’y voit entravé dans un "système de stéréotypes représentatifs" (Marin, "Dégénérescence” 41). Pour Marin nous sommes en pleine régression: "Le "Monde fantastique' est le retour de la réalité mais sous sa forme régressive et hallucinatoire" (Marin "Dégénérescence" 50). Que penser de cette “déconstruction sémiotique, idéologique et politique” (Vert 54) effectuée par Louis Marin? Elle donne froid dans le dos lorsqu'on la rapproche des zones neutres défonctionnalisées, que Foucault appelait bétérotopies, "sorte de contre-emplacements, ... les foires qui occupent les zones intermédiaires en bordure des villes et, plus encore, ces villages de vacances, cet ailleurs neutralisé où le citadin, l'espace d'un congé, replonge aux sources fantasmées d'une nudité primitive et éternelle” (Foucault 752-62). Marc Augé prendra le relais avec sa notion de non-lieu dont il constate la prolifération tous azimuts, un endroit inhabitable 
car il ne peut se définir "ni comme identitaire, ni comme relationnel, ni comme historique" (Augé 100). Même "façadisme” et "imagineering" à Legoland parc à thème créé par la société Lego au Danemark à Billund en 1968 et qui fut copié dans cinq autres sites au monde, tous dotés d’une zone Miniland composée de monuments construits en lego, et à Mini-Europe à Bruxelles, qui offre une voyage à travers le vieux Continent à échelle réduite, même pastiche, leurre, storytelling privé de toute dimension imaginaire. Tandis que la propriété de feu Michael Jackson, Neverland Ranch, avec son allusion à l'univers de Peter Pan, est sans doute l'emblème par excellence de la dissipation de l'imaginaire dans une fictionnalisation généralisée.

Les photomontages de Pierre Guimond, artiste québécois (fig. 2), quant à eux, déconstruisent ce que Baudrillard appelait déjà l'“utopie réalisée” (Baudrillard 76) en parlant des États-Unis. Car ce bout-à-bout de clichés oblige à reconsidérer notre approche du rêve américain. Guimond fait des États-Unis un énorme Disneyland où le concept même de tourisme devient caduc: ce que nous venons visiter n'existe pas. Le dépaysement, et la prise de distance critique qu'il suppose, n'est d'ailleurs plus possible en Amérique à en croire Baudrillard: "Quand vous vous retournez, l'Europe a tout simplement disparu” (Baudrillard 32). Si le seul choix qui nous reste—comme semblent le suggérer Guimond et Baudrillard—est d'entrer dans la fiction américaine, ils s'emploient tous deux à démontrer que cette fiction n'est plus étayée par l'imaginaire: “Or, la fiction n'est pas l'imaginaire. C'est ce qui anticipe sur l'imaginaire en le réalisant. ... Le mode de vie américain, lui, est spontanément fictionnel, puisqu'il est outrepassement de l'imaginaire dans la réalité" (Baudrillard 93). Le problème que Baudrillard entrevoit dans cette fiction, dans ce "gigantesque hologramme" (Baudrillard 33), dans cette utopie réalisée, c'est l'impasse dans laquelle se trouve une société où tout est disponible. Le film de Sofia Coppola Somewhere (2010) étale en images cette vacuité d'une existence qui dispose de tout, richesse, succès, et cetera. Selon Baudrillard, la seule issue s'avère la mort dont témoigne l'ambiance sépulcrale reflétée dans l'architecture : les baies vitrées "comme le cercueil de Blanche-Neige" (Baudrillard 34). Le réquisitoire de Baudrillard va même plus loin que le désenchantement de Marin: "La Californie n'a rien inventé: elle a tout pris à l'Europe, et le lui a resservi défiguré, privé de sens, repeint aux dorures de Disneyland” (Baudrillard 100). 
Toute ville imaginaire et ses dérives kitsch (et dès lors totalitaires par rapport à la liberté de conscience) se heurte à une aporie qui entame sa pérennité. Elle sera toujours en porte à faux par rapport à l'expérience du réel, le lieu de la balade et de la flânerie car elle a pour condition de possibilité "un oubli et une méconnaissance des pratiques. ... [D]e l'obscur entrelacs des conduites journalières" comme dit Michel de Certeau (Certeau 142).

Aragon dans Le Paysan de Paris (1926), arpente la ville, et en particulier le Passage de l’Opéra, y déambule au gré d'un itinéraire aléatoire jusqu'à s'y égarer. Il n'appréhende pas la ville en plongée, tel un panorama, mais frontalement. C'est un Paris labyrinthique et initiatique peuplé de "sphinx méconnus" (Aragon 20) qu'Aragon parcourt, se livrant, au détour d'une rue, au hasard objectif de la rencontre fortuite. La lueur glauque du Passage de l’Opéra lui prodigue pour un moment encore—car le boulevard Haussmann le menace_-ce paysage fantomatique des plaisirs et des professions maudites" (Aragon 21).

En effet, plus le lieu est équivoque, plus il est fécond en anecdotes, plus il stimule l'affabulation. La loge vitrée du concierge du Passage enferme deux petits vieillards astreints à ce "lieu absurde" (Aragon 27). Il n'empêche qu'Aragon leur prête “ces magnifiques dérèglements de l'imagination qu'on ne prête guère qu'aux poètes" (Aragon 28). Les concierges, péripatéticiennes ou petits commerçants, s'opposent ainsi au pouvoir impérial et capitaliste qui exerce sa suprématie par l'urbanisme haussmannien, ce grand éventreur qui exproprie en même temps les habitants et l'imaginaire des lieux. Pour Certeau—et Aragon ne l'aurait aucunement démenti-la marche est en revanche "un procès d'appropriation du système topographique par le piéton" (Certeau 148) car le geste cheminatoire non seulement actualise les possibilités de l'ordre spatial mais improvise, crée du discontinu et de l'équivoque dans les organisations panoptiques, transforme son ontologie en manières d'être et manières de faire, en singularités et en idiolectes, bref, en rêve: l'usager de la marche “voue certains lieux à l'inertie ou à l'évanouissement et, avec d'autres, il compose des tournures spatiales rares, accidentelles ou illégitimes" (Certeau 149).

Gilles Deleuze, dans L'Image-mouvement, remarque la même évolution d'une ville vue d'en haut à une ville "horizontale ou à hauteur d'homme" dans le passage du cinéma hollywoodien, d'action, à un cinéma qui a remplacé l'action ou la situation sensori-motrice par la balade urbaine. Il ajoute que celle-ci se fait "dans un espace quelconque, ... tissu dédifférencié de la ville” (Deleuze 279). Or, Aragon ou Breton utilisent certains 
quartiers de Paris, souvent “quelconques,"“'neutres" pour y puiser des épiphanies inexistantes avant le parcours lui-même.

Tout le roman d'Aragon est ainsi attiré vers cette force infinie de l'irréel, ce merveilleux quotidien que Perec appellera l'infra-ordinaire (Perec) et qui ouvre la ville à une appréhension anti-bourgeoise, non marchande, socialement et économiquement improductive. Car il y aura toujours un reste irréductible, le réel brut et mat: “Tu n’as pas dénombré les cailloux, les chaises abandonnées. Les traces de foutre sur les brins d’herbe. Les brins d'herbe" (Aragon 221).

La ville résistera toujours. Comme si elle était antithétique du mythe, comme si elle avait besoin du trivial. Le mythe comme image globale, configuration perçue par une vision surplombante, disparait pour laisser la place à un nouvel imaginaire, celui du passage, avec ses micro-récits, celui de la proximité, avec ses énigmes, ses épiphanies, son génie du lieu, avec ses nouvelles prosopopées, ville vue au raz du sol, depuis la loge des concierges, invitant à une approche plus ethnologique, éthologique. Julien Gracq, ayant subi le magnétisme de la ville de Nantes précisément parce qu'elle lui était partiellement interdite en raison de sa réclusion à l'internat, a prodigieusement fait comprendre que la ville imaginaire doit se nourrir de l'imaginaire d'une ville sous peine de se figer en fiction aseptisée. D’une part, notre image mentale d'une ville, sédimentée dans la mémoire, surgit de nos vagabondages quotidiens, du "pelotonnement irrégulier" (Gracq 2) causé par le lacis de nos allées et venues dans celle-ci. D’autre part, notre vision correspond rarement à l'agencement urbain officiel: "L'image qu'on garde d'une ville un moment habitée se développe, par une prolifération anarchique, à partir d'une cellule germinale qui ne coïncide pas forcément avec son 'centre', nerveux ou fonctionnel” (Gracq 28).

\section{CONCLUSION}

Avec Google Earth, Street View et la surveillance généralisée, cette velléité de la pratique de la ville risque également de disparaitre mais notre imaginaire sera toujours sollicité pour préserver une marge de non exploré, de recoins secrets mais signifiants, séduit peut-être par la démarche de Pierre Senges qui dans Ruines de Rome raconte l'histoire d'un employé du cadastre qui prépare une apocalypse végétale, un travail de sape botanique: “il sème un peu partout graines de plantes et mauvaises herbes qui, en s'épanouissant, percent le goudron, soulèvent le bitume, fissurent les murs," sa fronde ou sa mutinerie jardinière ayant pour fin de "lever les 
derniers pavés de la ville" (Senges 91), de parasiter l'architecture afin de créer un "paradis à l'échelle urbaine" (Senges 135). Ce lent travail de sape est non seulement pain béni mais un réel défi pour tout théoricien qui s'intéresse à la topoanalyse, à l’imaginaire des lieux et au dépaysement, aux passerelles entre "géographie du 'réel' et géographie de l'imaginaire”' (Westphal 275), car on oublie trop souvent que la ville, de plus en plus investie par les textes et les images, peut elle-même être saisie "par et dans les textes, par et dans les images"(Westphal 17) en fonction de la distance avec laquelle notre imaginaire l'appréhende. Ce n'est qu’à ce prix que l'imaginaire d'une ville émerge, non pas la fiction kitsch ou du façadisme que Walter Benjamin qualifierait déjà de "fantasmagorie" (Benjamin 47) au sens d’illusion mercantile aliénante, mais l'imaginaire de Bachelard, "l'imaginaire vif, mobile, fécond et déformant, qui maintient l'imagination béante" (Bachelard 8).

\section{SOURCES CITÉES}

Apollinaire, Guillaume. "La tour Eiffel”.Calligrammes. 1918. Paris: Mercure de France, 1925.

Aragon, Louis. Le Paysan de Paris. 1926. Paris: Gallimard, 1953.

Augé, Marc. Non-lieux. Paris: Editions du Seuil, 1992.

Bachelard, Gaston. L'Air et les songes. Paris: José Corti, 1943.

Bajac, Quentin and Didier Ottinger. Dreamlands: des parcs d'attraction aux cités du futur. Paris: Centre Pompidou, 2010.

Barrie, James Matthew; Peter Pan; or, the Boy Who Wouldn't Grow Up, 1911.

Battistini, Matilde. Simboli e allegorie. Milan: Mondadori Electa, 2002.

Baudrillard, Jean. Amérique, Paris: Bernard Grasset, 1986.

Benjamin, Walter. "Paris, die Hauptstadt des XIX. Jahrhunderts (1935)". In Illuminationen. Éd. Siegfried Unseld. Frankfurt am Main: Suhrkamp Verlag, 1961.185-200.Français:"Paris, capitale du XIXe siècle”. In Paris, capitale du XIXe siècle: le livre des passages. Trad. Jean Lacoste. Paris: Éditions du Cerf, 1989, pp. 47-59.Breton, André. La Clé des champs. Paris: Sagittaire, 1953.

Calvino, Italo. Le Città invisibili. Turin: Einaudi, 1972.Français: Les Villes invisibles. Trad. Jean Thibaudeau. Paris: Éditions du Seuil, 1974.

Chateaubriand, René. Vyage en Italie.1804. Genève: Droz, 1969.

Certeau, Michel de. L'Invention du quotidien, tome 1: Arts de faire.1980. Paris: Gallimard, 1990.

Coppola, Sonja. Somewhere, 2011. 
Debray, Régis. Contre-Venise. Paris: Gallimard, 1995.

—.Éloge des frontières. Paris: Gallimard, 2010.

Deleuze, Gilles. Cinéma 1. L’image-mouvement. Paris: Éditions de Minuit, 1983.

Derrida, Jacques. De l'hospitalité (Anne Dufourmantelle invite Jacques Derrida à répondre de l'hospitalité).Paris: Calman-Levy, 1997.

Eco, Umberto. Lector in fabula., Milano : Bompiani, 1979

Foucault,Michel. “Des espaces autres”. 1967.Dits et écrits 4. Paris: Gallimard, 1994. 752-62.

Gracq, Julien.La Forme d’une ville. Paris: José Corti, 1983.

Harvey, David. Spaces of Hope. Édimbourg: Edinburgh UP, 2000. 156-89. Français: “L’Espace urbain après le capitalisme: une utopie nécessaire".Trad. StathisKouvélakis. http://www.marxau21.fr/index.php?option=com_content\&view=article\&id=40:lespace-urbain-apres-lecapitalisme-une-utopie-necessaire\&catid=68:geographie-et-urbanisme\&Itemid=91 [consulté le 20 novembre 2013]

Hergé. Le Sceptre d'Ottokar, Bruxelles : Casterman, 1947.

Hugo, Victor. Notre-Dame de Paris.1831.Paris: Gallimard, 1975.

Koolhaas, Rem. Delirious NewYork: A Retroactive Manifesto for Manhattan. Londres: Academy Editions, 1978. Français: New York Délire. Trad. C. Collet. Marseille: Parenthèses, 2002.Lang, Fritz. Métropolis, 1927.

Marin, Louis. Le Portrait du roi. Paris: Éditions de Minuit, 1981.

—. "Dégénérescence utopique: Disneyland". Le Pouvoir dans ses représentations. Paris: Institut National d'Histoire de l'Art, 2008.

Morand, Paul. Fonquet ou Le Soleil offusqué. Paris: Gallimard, 1961.

Perec, Georges. L’infra-ordinaire. Paris : Seuil, 1995.

Queneau, Raymond. Zazie dans le métro. Paris: Gallimard, 1959.

Rimbaud, Arthur. “L’Orgie parisienne ou Paris se repeuple”1871. Poésies. Wikisource, 2012. http://fr.wikisource.org/wiki/L’Orgie parisienne/Édition Vanier 1895 [consulté le 30 novembre 2013].

Roelens, Nathalie. “Calvino e le “città metafisiche” di Giorgio de Chirico.”Italies, 2012, 16.“La plume et le crayon. Calvino, l'écriture, le dessin, l’image": 371-86.

Saint Augustin, Tractatus in Johannis evangelium. Paris: AELF, 1980.

Schifano, Jean-Noël. Naples. Paris: Éditions du Seuil, 1981.

—.Dictionnaire amoureux de Naples. Paris: Plon, 2007.

Saint Jean, Apocalypse, 21, 9-27 http://fr.wikisource.org/wiki/Apocalypse [consulté le 30 novembre 2013].

Senges, Pierre. Ruines-de-Rome. Paris: Éditions du Seuil, 2002.

Swift, Jonathan, Gulliver's Travels, 1927.

Tati, Jacques, Playtime, 1967. 
Trousson, Raymond. Voyages aux pays de nulle part. Histoire littéraire de la pensée utopique. Bruxelles: Université Libre de Bruxelles, 1979.

—."La Cité, l'architecture et les arts en utopie”. Revue littéraire en ligne 20 (2005):http://www.bon-atirer.com/volume20/rt.html[consulté 20 novembre 2013]

Vert, Xavier. “Louis Marin en Utopie: fiction, idéologie et représentation”. Louis Marin, Le pouvoir dans ses représentations. Paris: Institut national d'histoire de l'art, 2008, 53-75.

Westphal, Bertrand. La Géocritique. Réel, fiction, espace. Paris: Éditions de Minuit, 2007. 